\title{
MACULAR THICKNESS CHANGE AFTER PHACOEMULSIFICATION
}

\author{
A. Sirisha1, J. M. Lokabhi Reddy²
}

1 Post Graduate, Department of Ophthalmology, Sarojini Devi Eye Hospital, Hyderabad.

${ }^{2}$ Associate Professor, Department of Ophthalmology, Sarojini Devi Eye Hospital, Hyderabad.

\section{ABSTRACT}

Phacoemulsification for Cataract is the standard surgery in recent times with very good results and least complications. Macular area is the most important part of the Retina, which is very sensitive for any type of Insults on the Eye. It takes different durations for the Macula to return to normal condition after Phacoemulsification. Our aim of this study is to evaluate the macular thickness changes occurring after Phacoemulsification in post-operative period by using OCT in measuring the Retinal thickness.

\section{KEYWORDS}

Macular Thickness, OCT, Foveal Central Thickness, Phacoemulsification.

HOW TO CITE THIS ARTICLE: Sirisha A, Reddy JML. Macular thickness change after phacoemulsification. J. Evolution Med. Dent. Sci. 2016;5(19):982-985, DOI: 10.14260/jemds/2016/227

\section{INTRODUCTION \\ METHODOLOGY}

Prospective Observational Study.

Study Population: Patients diagnosed with immature senile cataracts who presented to Sarojini Devi Eye Hospital.

Sample size 50 eyes.

\section{Exclusion Criteria}

Any ocular diseases that might influence macular thickness such as history of Uveitis, Diabetic Retinopathy, Age-related macular degeneration, Intraoperative complications and inferior quality measurements of OCT due to media opacities. Examination was carried out including BCVA, Slit Lamp Biomicroscopy, Fundus examination and relevant general laboratory tests. In all selected cases Macular Thickness was studied with pre-op OCT and post-op OCT on first day, 7th day, 2 weeks, 4 weeks and 6 weeks. Informed consent was obtained from all the patients prior to their inclusion in the study.

\section{OCT Procedure}

Production and Display of Image: On Z axis, 1024 points are captured over a $2 \mathrm{~mm}$ depth to create a tissue density profile with resolution of $10 \mu$. On X-Y axis. The retinal map was divided into central point thickness, central $1 \mathrm{~mm}$ subfield and 2 peripheral ring areas with 3 and $6 \mathrm{~mm}$ diameter.

\section{RESULTS}

Optical coherence tomography is a non-contact, non-invasive technique that allows frequent measurements of the retinal thickness and is well tolerated by the patients. It has a high degree of reproducibility and repeatability. SLO/OCT generally has a good reproducibility profile. It was also concluded that in contrast to Fluorescein angiography, OCT quantifies retinal thickness in patients with cystoid macular oedema.[1] This study was done with a sample of 50 patients who had cataracts and underwent uneventful Phacoemulsification cataract surgery.

Financial or Other, Competing Interest: None.

Submission 01-02-2016, Peer Review 14-02-2016,

Acceptance 19-02-2016, Published 07-03-2016.

Corresponding Author:

Dr. J. M. Lokabhi Reddy,

\#66, Shanti Nagar, Masab Tank,

Hyderabad-500028.

E-mail:lokabhi@gmail.com

DOI: $10.14260 /$ jemds/2016/227
Initially, all the selected patients were examined clinically, i.e. with visual acuity, complete slit lamp examination, routine investigations like B.P. and R.B.S. All the selected cases were studied for Macular pathology and Thickness measurements pre-operatively and postoperatively on the days 1 and 7 and weeks 4th and 6th to evaluate any macular thickness changes in all the 9 areas within $6 \mathrm{~mm}$, i.e. Central foveal thickness, Inner superior, Inner nasal, Inner inferior, Inner temporal, Outer superior, Outer nasal, Outer inferior and Outer temporal quadrants.

The various associations of age, gender, HTN, DM were analysed in relation to the clinical findings and OCT findings. Out of the 50 cases studied, Males constituted 20 cases accounting to $40 \%$ and 30 cases $(60 \%)$ were Female. The patients fell in the age group of $49-75$ years. Out of the 50 cases, 7 patients were Diabetics, 6 Hypertensives and 4 patients had both DM and HTN (All are well controlled, without Retinopathy), 33 of them have no significant medical history.

A study done by Giansanti F, Bitossi A.[2] in September 2013, evaluated central Macular Thickness after Cataract Surgery in four groups of patients with Epiretinal membrane, patients with high myopia, patients with diabetes without retinopathy and healthy subjects. It was observed that increase in CMT was seen in patients from day 30 in patients with Epiretinal membrane and diabetes, reaching its maximum thickness on day 60 .

\begin{tabular}{|c|c|}
\hline $\begin{array}{c}\text { Retinal Thickness Change } \\
\text { Central Foveal Thickness }\end{array}$ & No. of Cases \\
\hline No Change & 8 \\
\hline Decrease & 4 \\
\hline $1-5$ um & 22 \\
\hline $5-10$ & 4 \\
\hline $10-20$ & 10 \\
\hline$>20$ um & 2 \\
\hline Table 1: Central Foveal Thickness \\
\hline
\end{tabular}

Pre-operatively, the central foveal thickness ranged from 206-310 microns.

Post-operatively, Central foveal thickness as per OCT after 6 weeks ranged from 207-328 microns. 


\begin{tabular}{|c|c|}
\hline $\begin{array}{c}\text { Retinal Thickness Change } \\
\text { Inner Superior }\end{array}$ & No. of Cases \\
\hline No Change & 19 \\
\hline Decrease & 9 \\
\hline $1-5$ um & 14 \\
\hline $5-10$ um & 6 \\
\hline $10-20$ um & 1 \\
\hline$>20$ um & 1 \\
\hline Table 2: Inner Superior Thickness \\
\hline
\end{tabular}

Pre-operatively, inner superior thickness ranged from 227-329 microns for the study group.

Post-operative OCT after 6 weeks of surgery gave results in the range of 240-335 microns.

\begin{tabular}{|c|c|}
\hline $\begin{array}{c}\text { Retinal Thickness Change } \\
\text { Inner Nasal }\end{array}$ & No. of Cases \\
\hline No Change & 11 \\
\hline Decrease & 15 \\
\hline $0-5$ um & 11 \\
\hline $5-10$ um & 5 \\
\hline $10-20$ um & 6 \\
\hline$>20$ um & 2 \\
\hline \multicolumn{2}{|c|}{ Table 3: Inner Nasal } \\
\hline
\end{tabular}

Pre-operatively, the Inner Nasal area thickness ranged from 213-378 microns.

After 6 weeks of surgery, the thickness ranged from 215378 microns.

\begin{tabular}{|c|c|}
\hline $\begin{array}{c}\text { Retinal Thickness Change } \\
\text { Inner Inferior }\end{array}$ & No. of Cases \\
\hline No Change & 17 \\
\hline Decrease & 12 \\
\hline $1-5$ um & 16 \\
\hline $5-10$ um & 4 \\
\hline $10-20$ um & 0 \\
\hline$>20$ um & 1 \\
\hline Table 4: Inner Inferior \\
\hline
\end{tabular}

In the present study, pre-operative Inner Inferior area thickness was 216-312 microns.

After 6 weeks of surgery, the thickness ranged from 215-455 microns.

\begin{tabular}{|c|c|}
\hline $\begin{array}{c}\text { Retinal Thickness Change } \\
\text { Inner Temporal }\end{array}$ & No. of Cases \\
\hline No Change & 10 \\
\hline Decrease & 12 \\
\hline $1-5$ um & 22 \\
\hline $5-10$ um & 5 \\
\hline $10-20$ um & 1 \\
\hline$>20$ um & 0 \\
\hline \multicolumn{2}{|c|}{ Table 5: Inner Temporal } \\
\hline
\end{tabular}

In the present study, preoperatively the thickness ranged from 264-327 microns.

After 6 weeks of surgery, the measured thickness ranged from 265-327 microns.

\begin{tabular}{|c|c|}
\hline $\begin{array}{c}\text { Retinal Thickness Change } \\
\text { Outer Superior }\end{array}$ & No. of Cases \\
\hline No Change & 13 \\
\hline Decrease & 10 \\
\hline $1-5$ um & 16 \\
\hline $5-10$ um & 7 \\
\hline $10-20$ um & 1 \\
\hline$>20$ um & 3 \\
\hline \multicolumn{2}{|c|}{ Table 6: Outer Superior } \\
\hline
\end{tabular}

Pre-operatively, in the present study the measurements ranged from 199-295 microns.

After 6 weeks of surgery, the measured range was 225305 microns.

\begin{tabular}{|c|c|}
\hline $\begin{array}{c}\text { Retinal Thickness Change } \\
\text { Outer Nasal }\end{array}$ & No. of Cases \\
\hline No Change & 12 \\
\hline Decrease & 7 \\
\hline $1-5$ um & 18 \\
\hline $5-10$ um & 9 \\
\hline $10-20$ um & 3 \\
\hline$>20$ um & 1 \\
\hline \multicolumn{2}{|r|}{ Table 7: Outer Nasal } \\
\hline
\end{tabular}

Pre-operative measurements ranged from 241-320 microns.

Post-operative measurements ranged from 242-329 microns.

\begin{tabular}{|c|c|}
\hline $\begin{array}{c}\text { Retinal Thickness Change } \\
\text { Outer Inferior }\end{array}$ & No. of Cases \\
\hline No Change & 18 \\
\hline Decrease & 7 \\
\hline $1-5$ um & 19 \\
\hline $5-10$ um & 4 \\
\hline $10-20$ um & 0 \\
\hline$>20$ um & 2 \\
\hline \multicolumn{2}{|c|}{ Table 8: Outer Inferior } \\
\hline
\end{tabular}

Pre-operative measurements ranged from 244-314 microns.

Post-operatively, after 6 weeks of surgery the measurements ranged between 240-314 microns.

\begin{tabular}{|c|c|}
\hline $\begin{array}{c}\text { Retinal Thickness } \\
\text { Change Outer Temporal }\end{array}$ & No. of Cases \\
\hline No Change & 17 \\
\hline Decrease & 10 \\
\hline $1-5$ um & 16 \\
\hline $5-10$ um & 5 \\
\hline $10-20$ um & 1 \\
\hline$>20$ um & 1 \\
\hline \multicolumn{2}{|c|}{ Table 9: Outer Temporal } \\
\hline
\end{tabular}

Pre-operative measurements ranged from 237-286 microns.

Post-operatively, 6 weeks after the surgery the measurements are 225-295 microns.

\section{DISCUSSION}

The present study showed central foveal thickness reducing post-operatively from 1 st week to 6 th week in 13 of the cases and OCT measurements reduced from preoperative to 4 th post-operative OCT that was done after 6 weeks of surgery.

Gerasimos, Georgopoulos.[3] et al. studied foveal thickness after Phacoemulsification as measured by OCT in Aukland, New Zealand. It was a prospective study of 79 eyes assessed by OCT on day 1 and weeks 2 and 4 after uncomplicated Phacoemulsification. The thickness of the retina is significantly smaller than the thickness of the retina on day 1 and week 2 . It returned to the initial levels on week 4 . Based on previous research (Biro, et al. 2008).[4] it was hypothesized that the foveal thickness would be increased postoperatively. Regarding hypothesized relation between the amount of Phacoemulsification energy and the foveal 
thickness, no significant correlation was detected.

According to a Chinese study by Cheng.[5] et al. in 2002 showed higher Phacoemulsification power groups had increased foveal thickness compared to lower Phacoemulsification power groups in week 1. Perente.[6] et al. in 2007 evaluated macular thickness changes after uncomplicated Phacoemulsification surgery. They have concluded that statistically increase in macular thickness was detected at post-operative early periods, i.e. the first week after operation. The increase in macular thickness starts from Parafoveal regions. Longer follow-up of patients is required to know the macular consequences.

Macular oedema is the result of accumulation of fluid in the retinal layers around the fovea. It contributes to vision loss by altering the functional cell relationship in the retina and promoting an inflammatory preparative response. Macular oedema may be intracellular or extracellular. Intracellular accumulation of fluid is also called cytotoxic oedema. It is an alteration of the cellular ionic distribution. Extracellular accumulation of the fluid, which is more frequent and clinically more relevant is directly associated with an alteration of blood retinal barrier. The following parameters are relevant for clinical evaluation of macular oedema.

1. Extent of the macular oedema.

2. Distribution of the oedema in the different macular areas.

3. Central foveal involvement.

4. Fluorescein leakage and intraretinal cysts.

5. Chronicity of the oedema, i.e. time elapsed since initial diagnosis and response to therapy.

In the present study, there were 7 diabetics among which 4 showed increase in macular thickness post-operatively especially in inner nasal, inner inferior and central foveal areas. A study conducted by Soon II Kwon.[7] et al. in August 2011 showed macular oedema in $18 \%$ of the patients with diabetes who underwent Phacoemulsification surgery.

In the present study, there were six hypertensives among 50 of the patients studied and 2 of them showed minor increase in macular thickness post-operatively. The increased measurements were within the normal range. One among them had thickness coming back to pre-operative levels by the sixth week of post-operative period.

There were 4 patients having both diabetes and hypertension in the present study. Two patients showed increase in the thickness, which remained high even after 6 weeks of post-operative period. It was concluded by Kecic D, et al.[8] in 2009, that there is a significant increase in macular parameters after uneventful Phacoemulsification in patients having both diabetes and hypertension. The study was conducted on 87 patients. The findings of the present study corroborate with the above findings.

\section{CONCLUSIONS AND SUMMARY}

Despite comprising a mere $0.1 \%$ of total body surface and only $0.27 \%$ of the anterior body surface, the contribution of eyes and their significance to the individuals and the society is exceptionally of high magnitude. Cataract, leading to vision impairment significantly affects the functioning of the individual and brings about major lifestyle changes.

In ophthalmological field, cataract surgery has undergone rapid changes in the recent decades. Small incision cataract surgery, especially by Phacoemulsification has emerged over the years. Phaco has now captured the centre stage in the surgical management of cataract.

It has revolutionized the management of cataract surgery and has facilitated achieving new peaks in quality of surgery and recovery of visual acuity to pre-cataract stage.

The risk of visually significant CME has decreased with the development of advanced surgical techniques, such as modern Phacoemulsification and foldable intraocular lenses. The risk of CME formation after cataract surgery may increase in the presence of several factors, such as advancing age and diabetes. Cataract surgery in diabetic patients may accelerate pre-existing macular oedema. Even in the absence of diabetic macular oedema, diabetic patients tend to have a higher risk of developing macular oedema after uncomplicated Phacoemulsification.

OCT can be used quantitatively and qualitatively to follow retinal thickness over time. The advantages of OCT over other imaging modalities available include its non-invasive approach, quick imaging acquisition and safety profile. Since the introduction of OCT, the diagnosis and follow-up of macular oedema has been greatly facilitated. This modality offers a non-invasive imaging technique that provides high resolution cross-sectional images of macula. OCT produces highly reproducible measurements, so that serial examination may be used for follow-up. OCT is extremely valuable in following a patient's response to treatment.

The present study did not reveal any relevance to gender in the development of macular oedema after uncomplicated Phacoemulsification. Mild increased macular thickness was observed in 6 among 20 Males and 9 among 30 Females in the postoperative period that accounts to $30 \%$ in each of the sexes. Hypertension alone does not seem to affect macular thickness significantly in the post-operative period.

Diabetes and hypertension when coexisted can greatly influence development of macular oedema irrespective of age or gender. Among 4 cases having both DM and HTN, 2 patients accounting to $50 \%$ have showed considerable increase in macular thickness post-operatively.

Clearly, the future of OCT imaging is bright. The detail with which this technology allows the Ophthalmologist to become familiar with anatomic alterations of the pathologic retina is nothing short of marvellous. This greatly enables to plan individual patient's management and to achieve better visual outcome.

\section{REFERENCES}

1. Massin P, Girach A, Erginay A, et al. OCT-a key to the future management of patients with diabetic macular edema. Acta ophthalmol scand 2006;84(4):466-74.

2. Giansanti F, Bitossi A, Giacomelli G, et al. Evaluation of macular thickness after uncomplicated cataract surgery using OCT. European Journal of Ophthalmology 2013;23(5):751-6.

3. Gerasimos Th Georgopoulos. Foveal thickness after phacoemulsification as measured by optical coherence tomography. Clin Ophthalmol 2008;2(4):817-820.

4. Biro Z, Balla Z, Kovacs B. Change of foveal and perifoveal thickness measured by OCT after phacoemulsification and IOL implantation. Eye (Lond) 2008;22(1):8-12.

5. Cheng B, Liu Y, Liu X, et al. Macular image changes of optical coherence tomography after phacoemulsification. Zhonghua Yan Ke Za Zhi 2002;38(5):265-7. 
6. Perente I, Utine CA, Ozturker C, et al. Evaluation of macular changes after uncomplicated phacoemulsification surgery by optical coherence tomography. Curr Eye Res 2007;32:241-247.

7. Soon Il Kwon, Duck JH, Seo JY, et al. Evaluation of changes of macular thickness in diabetic retinopathy after cataract surgery. korean J Ophthalmol 2011;25(4):238-242.
8. Kecik D, Makowiec TM, Golebiewska J, et al. Macular thickness and volume after uncomplicated phacoemulsification surgery evaluated by optical coherence tomography. A one-year follow-up. Neuro endocrinol lett 2009;30(5):610-4. 